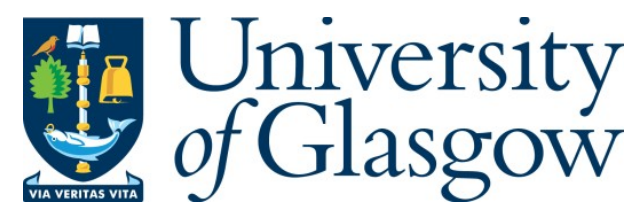

Kennedy, P. G.E., Graner, M. W., Walker, D., Pointon, T., Fringuello, A. and Yu, X. (2020) Recombinant antibodies derived from laser captured single plasma cells of multiple sclerosis brain identified phage peptides which may be used as tools for characterizing intrathecal IgG response. Journal of Neuroimmunology, 347, 577319.

(doi: 10.1016/j.jneuroim.2020.577319)

This is the Author Accepted Manuscript.

There may be differences between this version and the published version. You are advised to consult the publisher's version if you wish to cite from it.

$\underline{\text { https://eprints.gla.ac.uk/220428/ }}$

Deposited on: 13 July 2020

Enlighten - Research publications by members of the University of Glasgow http://eprints.gla.ac.uk 


\title{
Recombinant antibodies derived from laser captured single plasma cells of multiple sclerosis brain identified phage peptides which may be used as tools for characterizing intrathecal IgG response
}

\author{
Peter GE Kennedy ${ }^{\mathrm{a}}$, Michael W Graner ${ }^{\mathrm{b}}$, Deandra Walker ${ }^{\mathrm{c}}$, Tiffany Pointon ${ }^{\mathrm{c}}$, Anthony \\ Fringuello ${ }^{\mathrm{b}}$, Xiaoli $\mathrm{Yu}^{\mathrm{b}} *$ \\ ${ }^{\text {a }}$ Institute of Infection, Immunity and Inflammation, University of Glasgow, Glasgow, UK, \\ ${ }^{\mathrm{b}}$ Department of Neurosurgery, University of Colorado Anschutz Medical Campus, Aurora, \\ Colorado, USA.
}

${ }^{c}$ Department of Neurology, University of Colorado Anschutz Medical Campus, Aurora, Colorado, USA.

*Corresponding author

E-mail: Xiaoli.Yu@CUanschutz.edu

Telephone: 303-724-9182

Running Title: MS brain antibodies selected phage peptides bind to intrathecal IgG

Key words: Multiple Sclerosis, lesion, laser capture microdissection, phage display, peptide, $\mathrm{IgG}$, cerebrospinal fluid (CSF), oligoclonal bands, recombinant antibody, intrathecal antibody 


\begin{abstract}
Oligoclonal bands and increased IgG antibody levels can be detected in the cerebrospinal fluid in vast majority of patients with Multiple Sclerosis (MS). However, the antigenic specificity of oligoclonal IgG has yet to be determined. Using laser capture microdissection, we isolated single CD38+ plasma cells from lesion areas in two autopsy MS brains, and generated three recombinant antibodies (rAbs) from clonally expanded plasma cells. Panning phage-displayed random peptide libraries was carried out to determine peptide antigen specificities of these MS brain rAbs. We identified 25 high affinity phage peptides from which 5 peptides are unique. Database searches revealed that they shared sequence homologies with Epstein-Barr nuclear antigens 4 and 6, as well as with other viral proteins. Significantly, these peptides were recognized by intrathecal IgG and oligoclonal IgG bands in other MS patients. Our results demonstrate that functional recombinant antibodies can be generated from clonally expanded plasma cells in MS brain lesions by laser capture microdissection, and that these MS brain rAbs have the potential for determining the targets of intrathecal $\mathrm{IgG}$ and oligoclonal bands.
\end{abstract}




\section{Introduction}

Multiple Sclerosis (MS) is a chronic demyelinating disease of the central nervous system (CNS) which is a major cause of neurological disability, especially in young adults, in northern temperate zones (Gilden, 2005, Kennedy and Steiner, 1994). Typical immunological features in MS patients include the presence of clonally expanded B-lymphocytes and plasma cells in the cerebrospinal fluid (CSF), an increase in CSF IgG, and the presence of oligoclonal bands in over $90 \%$ of cases (Owens et al. , 2003, Reiber et al. , 1998, Yu et al. , 2011a). While oligoclonal bands are known to be directed against specific pathogens in a number of chronic neurological infections such as subacute sclerosing panencephalitis (SSPE), cryptococcal meningitis, neuroborreliosis and neurosyphilis (Gilden, 2005), the antigenic specificity of oligoclonal IgG in MS, despite its critical importance in disease activities (Ferreira et al. , 2014, Joseph et al. , 2009), has so far proved to be elusive (Owens et al. , 2009). Our recent publication suggested that there is a complex relationship between oligoclonal bands and immunoglobulin G antibodies in MS (Beseler et al. , 2017).

By screening phage-displayed random peptide libraries with recombinant antibodies generated from clonally expanded B cells in MS CSF, we have demonstrated that high affinity linear peptides can be identified (Yu et al. , 2011b, Yu et al. , 2006b). Furthermore, we showed that peptides selected by MS CSF were recognized by intrathecal IgG antibodies in MS patients over time, and that the peptides can be used to investigate the disease progression (Yu, Burgoon, 2011a). In addition, we recently reported that in MS the number of oligoclonal IgG bands has no relationship with the concentration of CSF IgG (Beseler, Vollmer, 2017), suggesting that the increased IgG in MS may be represented by IgGcontaining immune complexes. Phage-displayed random peptide libraries provide a unique unbiased approach to identify high affinity targets regardless of previous knowledge.

We herein report the generation of recombinant antibodies derived from laser captured single plasma cells of MS brain lesions, and results of using these antibodies to determine which, if any, peptide antigens are specific to the intrathecal MS antibody response ( $\mathrm{Yu}$, Gilden, 2011b). Laser-capture microdissection (LCM) of clonally expanded plasma cells from Subacute sclerosing panencephalitis (SSPE) brain originally showed that overrepresented IgG sequences could be used to produce functional recombinant antibodies (rAbs) capable of 
recognising specific disease-related antigens (Burgoon et al. , 2005). Subsequent studies used 19 rAbs generated from such clonally expanded B cells and native IgG from the CSF of 3 MS patients, combined with the technique of panning with phage displayed random peptide libraries, to show antibody-peptide interactions shared by the rAbs and IgG obtained from the same MS patient (Yu, Burgoon, 2011a). Further, the same technological approach showed that the panned phage peptides bound specifically to IgG in the CSF of 5 MS patients over time (longitudinal sampling) (Yu, Burgoon, 2011a). This was significant because CSF oligoclonal IgG persists throughout the lifetime of MS patients (Petereit and Reske, 2005). In the present work, we generated MS brain recombinant antibodies from laser captured microdissection of clonally expanded single plasma cells and identified high affinity phage peptides using a similar panning approach. We have extended the range of the possible antigenic specificity of CSF IgG in MS by showing that phage peptides selected by MS brain-derived rAbs are recognized by intrathecal $\operatorname{IgG}$ and oligoclonal bands of other unrelated MS patients.

\section{Materials and Methods}

2.1. Generation of MS brain derived recombinant antibodies from single plasma cells by laser capture microdissection

Using established techniques for single-cell laser capture microdissection (LCM) (Burgoon, Keays, 2005), single CD38-positive plasma cells were isolated from multiple lesion areas of frozen autopsied samples from two MS brains. One brain (MS brain A: ST) was from the autopsy of a relapsing remitting MS (RRMS) patient. The second brain (MS brain B: G2) was from the autopsy of a secondary progressive MS (SPMS) patient, and which was reported previously to have meningeal follicles and EBV-infected B cells (Serafini et al. , 2004). Each brain sample contained expanded numbers of CD20-positive B lymphocytes and CD38positive plasma cells compared to control non-MS brain samples. Laser capture microdissection and following IgG heavy and light chain variable sequence amplification were performed on 40-50 individually micro-dissected plasma cells for each brain. The construction and generation of the recombinant antibodies (rAbs) utilized techniques as previously described (Burgoon, Keays, 2005).

Briefly, CD38+ plasma cells were identified by staining and micro dissected from $12 \mu \mathrm{m}$ brain sections onto individual caps. Cells were lysed with NP-40 and reverse-transcription 
performed on cap surfaces. Heavy chain and light chain antibody sequences were PCRamplified from each CD38+ cell, and subcloned into TA vector for sequencing. The selected pairs of heavy and light chain IgG sequences were subcloned into mammalian expression vectors and transfected into HEK293-EBNA cells, and secreted human recombinant antibodies ( $\mathrm{rAb}$ ) contained a flag epitope, were purified by Protein A affinity chromatography. The recombinant antibodies are named as A1 (ST-176), A2 (ST-196), and B1 (G2-160).

\subsection{Biopanning, phage titration and amplification}

The Phage Display Peptide Libraries (12mer + 7mer) (New England BioLabs, Beverly, MA) were used for affinity selection of specific peptides for the MS brain rAbs. The panning procedure was essentially as described (Yu et al. , 2006a). MS brain rAbs at a concentration of $150 \mu \mathrm{g} / \mathrm{ml}$ were added to wells of Reacti-Bind ${ }^{\mathrm{TM}}$ Protein A-coated clear strip plates (Thermo Scientific, Rockford, IL) in $50 \mu 1$ Tris-buffed saline (TBS) and incubated overnight at $4^{\circ} \mathrm{C}$. After blocking, the phage peptide library $\left(1.5 \times 10^{10} \mathrm{pfu}\right)$ in $100 \mu \mathrm{l}$ of TBST (TBS$0.5 \%$ Tween ${ }^{20}$ ) was added to the wells and incubated for $1 \mathrm{~h}$ at room temperature. Elution of bound phage was performed at either room temperature for $20 \mathrm{~min}$ or at $37^{\circ} \mathrm{C}$ for $10 \mathrm{~min}(\mathrm{Yu}$ et al. , 2009). Three to five rounds of panning were carried out. The affinity-selected phage were titered after each rounds of panning. Phage titering and amplification was as described (Yu, Gilden, 2006a).

\subsection{Single-point 96 well ELISA}

Primary single-point 96 well ELISA was carried out as reported (Yu, Owens, 2006b). Individual plaques selected by each antibody from the titer plates of second and third pans or higher pans were picked and placed into wells of U96 DeepWell ${ }^{\mathrm{TM}}$ plates (Nunc International, Rochester, NY) containing $500 \mu \mathrm{l}$ of a 1:10 dilution of optical density $=1.0$ or 1:100 dilution of overnight culture of E. coli ER 2738 in LB medium. The plate was covered with breathable sealing tape (NUNC). After $4.5 \mathrm{~h}$ of infection at $37{ }^{\circ} \mathrm{C}$ at $250 \mathrm{rpm}$, the DeepWell plates were centrifuged at $4,000 \mathrm{rpm}$ for $10 \mathrm{~min}$ at $4{ }^{\circ} \mathrm{C}$ in a Sorvall RT 6000 refrigerated centrifuge. The supernatants containing amplified phage were transferred to a new DeepWell plate and stored at $4^{\circ} \mathrm{C}$. For primary fast screening of potential positive phage, a microtiter plate (Corning, Corning, NY) was coated with $50 \mu 1$ of MS brain rAbs in $0.1 \mathrm{M}$ 
carbonate buffer, $\mathrm{pH} 9.5$ at $1 \mu \mathrm{g} / \mathrm{ml}$, blocked with 3\% BSA for $1 \mathrm{~h}$ and washed with TBST three times. Phage supernatants $(50 \mu \mathrm{l})$ from the DeepWell plate were added directly to individual wells and incubated for $1 \mathrm{~h}$ with shaking at room temperature. Plates were washed with TBST 10 times for 1 min each time, followed by incubation with $50 \mu$ of 1:500 dilution of horseradish peroxidase (HRP)/anti-M13 antibody conjugate (New England Biolabs) in 1\% BSA/Tween ${ }^{20}$ for $1 \mathrm{~h}$. The plates were washed 8 times with TBST. Bound phage were detected using HRP substrate ABTS (Vector Laboratories, Burlingame, CA). After incubation with substrate for 20-30 min, absorbance at $405 \mathrm{~nm}$ was determined spectrophotometrically with a Microplate Reader (Bio-Rad, Hercules, CA).

\subsection{ELISA determining phage binding specificity}

To confirm positive phage results from single-point 96-well ELISA, MS brain rAb (50 $\mu 1$ at 1 $\mu \mathrm{g} / \mathrm{ml}$ ) with human IgG antibody as negative control was coated in duplicate in wells of an ELISA plate overnight at $4{ }^{\circ} \mathrm{C}$. Wells were blocked with $3 \%$ BSA for $1 \mathrm{~h}$ and $5 \times 10^{9}$ corresponding phage were added to each well. After washing, bound phage were detected as described above. For dose-response ELISA, serial 2-fold phage dilutions were added to wells coated with respective panning MS brain $\mathrm{rAb}$ and a human $\mathrm{IgG}$ as negative control. Phage detection was as described above. ELISA was repeated at least once.

\subsection{Dose-response phage-mediated immuno-PCR (IPCR)}

Phage-mediated IPCR was performed as described (Yu et al. , 2007). Reacti-Bind ${ }^{\mathrm{TM}}$ wells of protein A-coated clear strip plates (Thermo Scientific, Rockford, IL) were coated with $50 \mu \mathrm{l}$ of CSF or serum ( $1 \mu \mathrm{g} / \mathrm{ml} \mathrm{IgG)} \mathrm{and} \mathrm{with} \mathrm{pre-immune} \mathrm{human} \mathrm{IgG} \mathrm{(Alpha} \mathrm{Diagnostic)} \mathrm{in} \mathrm{TBS}$ (50 $\mathrm{mM}$ Tris- $\mathrm{HCl}, 150 \mathrm{mM} \mathrm{NaCl}$ ) at room temperature for $2 \mathrm{~h}$, washed with TBS containing $0.05 \%$ Tween $^{20}$ (TBST) and blocked in 3\% non-fat dry milk/0.05\% TBST at room temperature for $1 \mathrm{~h}$. Serial 10-fold phage dilutions in duplicate were added to MS CSF/serum IgG-coated wells and incubated at room temperature for $2 \mathrm{~h}$. Bound phage were lysed in 50 $\mu \mathrm{l}$ of double-deionized water by heating the plates at $95{ }^{\circ} \mathrm{C}$ for $15 \mathrm{~min}$ to release singlestranded phage DNA as the template for real-time PCR in an Applied Biosystems 7500 Fast Real-Time PCR system (Applied Biosystems, Foster City, CA). For standard SYBR $®$ Green PCR, each reaction $(20 \mu \mathrm{l})$ consisted of $1 \times$ power SYBR ${ }^{\circledR}$ Green master mix (Applied Biosystems), $750 \mathrm{nM}$ of each M13 phage primer and $4 \mu \mathrm{l}$ of phage template. Thermal cycle 
conditions were $95{ }^{\circ} \mathrm{C}$ for $10 \mathrm{~min}$, followed by 40 cycles at $95{ }^{\circ} \mathrm{C}$ for $15 \mathrm{~s}$ and $60{ }^{\circ} \mathrm{C}$ for $45 \mathrm{~s}$. Fast real-time PCR was conducted using $1 \times$ Fast SYBR ${ }^{\circledR}$ Green master mix, with thermal cycling at $95{ }^{\circ} \mathrm{C}$ for $20 \mathrm{~s}$, followed by 40 cycles at $95{ }^{\circ} \mathrm{C}$ for $3 \mathrm{~s}$ and $60{ }^{\circ} \mathrm{C}$ for $30 \mathrm{~s}$. A control reaction without template was included in each run.

\subsection{Western blots}

NuPAGE Bis-Tris Mini Gels (Invitrogen, Carlsbad, CA) were used for phage SDS-PAGE analysis with $1 X$ MOPS SDS Running Buffer. Phage peptides $\left(1 \times 10^{11} /\right.$ well $)$ in TBS were denatured and reduced with $1 \mathrm{X}$ protein sample buffer containing $\beta$ mercaptoethanol (Pierce Biotechnology, Rockford, IL) at $95^{\circ} \mathrm{C}$ for $10 \mathrm{~min}$. Duplicate gels were eletrophoresed for 50 min under constant voltage of $200 \mathrm{~V}$. The gel was then electroblotted onto a PVDF membrane (Bio-Rad) for 60 min under constant voltage of $15 \mathrm{~V}$ using Trans-Blot ${ }^{\circledR}$ Semi-Dry Cell (Bio-Rad). After blocking with $1 \mathrm{X}$ casein/TBS (Vector Laboratories) containing $0.1 \%$ Tween ${ }^{20}$ for $1 \mathrm{~h}$, the membrane was incubated with corresponding MS brain rAb $(1 \mu \mathrm{g} / \mathrm{ml})$ for $1 \mathrm{~h}$ in $1 \mathrm{X}$ casein/TBS buffer with $0.1 \%$ Tween $^{20}$. Peroxidase conjugated goat anti-human $\operatorname{IgG}(\mathrm{H}+\mathrm{L})$ (Sigma, St. Louis, MO) at a dilution of 1:5000 was used to detect $\mathrm{rAb}$ binding to phage. After incubation with each antibody, membranes were washed once with TBST for 15 min, then four more times for 5 min each. The membrane was incubated with SuperSignal ${ }^{\circledR}$ West Femto Maximum Sensitivity chemiluminescent substrate (Pierce) as recommended by the manufacturer. For detection of phage pIII protein, duplicate membranes were incubated with a 1:25,000 dilution of anti-M13 pIII monoclonal antibody (New England BioLabs). A 1:25,000 dilution of goat anti-mouse IgG peroxidase conjugate (Vector Laboratories) was used as secondary antibody followed by chemiluminescent detection as described ( $\mathrm{Yu}$, Burgoon, 2011a).

\subsection{Isoelectric focusing (IEF) immunoblotting}

IEF immunoblotting was as described (Yu, Burgoon, 2011a). CSF (200-500 $\mu$ l) was centrifuged on an Amicon Ultra $0.5-\mathrm{ml} 30 \mathrm{~K}$ cellulose centrifugal filter unit at $14,000 \times \mathrm{g}$ for 30 min at room temperature before IEF using the SPIFE® IgG IEF kit (Helena Laboratories, Beaumont, TX) and a SPIFE 3000 electrophoresis analyzer. Wicks were soaked in an anode $(0.3 \mathrm{M}$ acetic acid) or cathode $(1 \mathrm{M} \mathrm{NaOH})$ solution and applied to the edge of a SPIFE® IgG IEF gel. Five microliters of MS CSF/sera [3-5 $\mu \mathrm{g}$ IgG for phage probe and $100 \mathrm{ng}$ IgG 
for alkaline phosphatase (AP)-conjugated anti-human IgG probe] were loaded into wells of an SPIFE IEF gel. After electrophoresis at $700 \mathrm{~V}$ for $1 \mathrm{~h}$ at $15{ }^{\circ} \mathrm{C}$, samples were transferred to PVDF membranes for $45 \mathrm{~min}$, followed by blocking in Helena blocking agent $(1 \mathrm{~g}$ bovine milk protein $/ 50 \mathrm{ml} 1 \times \mathrm{TBS}$ ) for $1 \mathrm{~h}$ at room temperature. Membranes were incubated with the respective phage peptide at concentrations ranging from $5.0 \times 10^{10}$ to $1.5 \times 10^{11} \mathrm{pfu} / \mathrm{ml}$ in $1: 10$ Helena blocking agent/TBST (blocking buffer) at room temperature for $2 \mathrm{~h}$. After washing with $0.05 \%$ Tween-TBS, membranes were incubated with mouse anti-M13 mAb at a 1:500 dilution in blocking buffer, followed by incubation with 1:500 dilution of AP conjugated antimouse $\operatorname{IgG}$ at room temperature for $1 \mathrm{~h}$. Membranes were developed with NBT/BCIP substrate. For control blots, membranes were incubated for $1 \mathrm{~h}$ with 1:1000 dilutions of APanti-human $\operatorname{IgG}(\mathrm{H}+\mathrm{L})$ in blocking buffer, followed by NBT/BCIP detection.

\subsection{DNA sequencing and peptide database search}

Single-stranded phage DNA was purified and sequenced to deduce the amino acid sequence of the peptide. All DNA sequencing was performed at the University of Colorado Cancer Centre Genomics and Microarray Shared Resource. Peptide sequences were deduced from DNA sequences. Consensus peptides were identified by sequence alignment using Clustal Omega (https://www.ebi.ac.uk/Tools/msa/clustalo/). For identifying candidate proteins, each peptide was used for the database search in BLAST (http://www.ncbi.nlm.nih.gov/) using Swiss-Prot protein sequences database.

\section{Results}

3.1. Generation of recombinant antibodies from clonally expanded single plasma cells by laser captured microdissection of MS plaques

The three MS brain rAbs used in this study were generated from clonal expanded CD38+ plasma cells isolated by single-cell laser microdissection from lesional areas of 2 MS brains (Burgoon, Keays, 2005). Each of the brain rAbs were from the sequences of expanded plasma populations (30-50 plasma cells) of the corresponding lesional tissues. One plaque tissue was from a RRMS brain (A, ST), and the other was from a SPMS brain (B, G2). 
Sequences of both the IgG heavy and light chain V regions were determined for populations of (CD38-positive) plasma cells after RNA isolation and RT-PCR. Multiple areas from each brain expressed repertoires of clonally expanded IgG sequences (data not shown). The CDR3 sequences of several expanded clones are shown in Table 1. The VH and VL CDR3 amino acid sequences, the closest germline segment, and the degree of homology are shown. Two rAbs were generated from MS Brain A named MS-A1 (ST-176), and MS-A2 (ST-196), and one $\mathrm{rAb}$ was generated from MS Brain B named MS-B1 (G2-160). It can be seen that the homology was always high (overall $87.5 \%-97.1 \%$ ) with the greatest homology shown by brain rAb MS-A1 (ST-176), and the lowest by brain rAb MS-B1 (G2-160) that show comparative somatic mutation from germline Ig alleles.

\subsection{High affinity phage peptides that reacted with $M S$ brain rAbs were identified by panning phage displayed random peptide libraries}

Three MS brain rAbs generated from clonally expanded plasma cells by LCM were studied for antigen specificity by panning combined phage-displayed random peptide libraries (Ph.D. $12+$ Ph.D. 7, 12-mers and 7-mers). Phage panning procedures were as described previously (Yu, Gilden, 2006a). A total of 5 pans were performed. We applied a high throughput approach for fast screening of phage peptides which were specific to panning MS brain rAbs using a streamlined protocol (Yu, Owens, 2006b). We identified 25 high affinity phage peptides by the 3 MS brain rAbs from which 5 peptides are unique. MS brain rAb A1 (ST176) identified 2 unique peptides, MS brain rAb A2 (ST-196) identified 2 unique peptides, and MS rAb B1 (G2-160) selected one unique peptide. We named the peptides based on the rAb used for panning. For example, phage peptide A2 (ST-196) D2 represents peptide D2 selected by MS rAb A2 (ST-196). We performed sequence alignment analysis and no common antigens were found (data not shown). The results of peptide sequences are listed in Table 2. Database searches revealed that they shared sequence homologies with Epstein-Barr nuclear antigens 4 and 6 , as well as with other viral proteins. In addition, these peptides show high sequence homologies with human proteins such as TRPM8 channel-associated factor 2, G-protein coupled receptor, and ubiquitin-like modifier-activating enzyme. Table 3 lists candidate proteins of the BLAST (https://blast.ncbi.nlm.nih.gov/Blast.cgi?PAGE=Proteins) searches using UniProtKB/Swiss-Prot (Swissprot) database for both human and virus proteins. 


\subsection{Binding specificities of selected phage peptides by MS brain recombinant antibodies}

ELISA was used to determine phage peptide binding specificities to panning MS brain rAbs (Yu, Gilden, 2006a). The results of these experiments are shown in Figure 1. It can be seen in Fig 1A that phage peptides selected by MS brain rAb A1(ST-176) have higher bindings to the panning MS brain rAb A1 (ST-176) but less to control human IgG. Fig. 1B shows that all phage clones selected bind specifically to the panning MS rAb A2 (ST-196) but not to control human IgG. In Fig 1C, we show the cross reactivity of selected phage peptides to nonpanning brain rAbs. It can be seen that phage peptides A1 (ST-176) C3 and A1 (ST-176) B1 identified by brain rAb A1 (ST-176) bind to panning rAb A1 (ST-176) as well as to nonpanning rAb A2 (ST-196) and control human IgG, suggesting that these peptides were polyspecific. Peptides A2 (ST-196) F4 and A2 (ST-196) D2 identified by MS brain rAb A2 (ST196) were specific only to panning MS rAb A2 (ST-196), but not to non-panning rAb A1 (ST-176) and control human IgG, suggesting these peptides were specific. We further characterized phage peptides A2 (ST-196) D2 and F4 (selected by MS brain rAb A2) with dose-response ELISA as shown in Fig. 1D. Both peptides bind to panning MS brain rAb A2 (ST-196) in a dose-responsive manner.

To further characterize these MS brain rAb specific peptides, we carried out Western blot analysis. Representative phage panned by 2 brain rAbs were separated on a gradient of 4 $12 \%$ SDS polyacrylamide gel under reducing and denaturing conditions, blotted to a PVDF membrane and incubated with corresponding brain rAbs. Phage peptides A2 (ST-196) F6, A2 (ST-196) C3, and B1 (G2-160) D2, B1 (G2-160) F2 were recognized by corresponding panning MS brain rAb A2 (ST-196) and B1 (G2-160), suggesting that these peptides were linear in conformation. Positive control was phage blot probed with anti-pIII antibody (Fig. 2).

3.4. Phage peptides selected by MS brain $r A b$ were recognized by intrathecal IgG from MS patients

To determine whether the MS brain rAb-selected peptides can be recognized by CSF IgG from other MS patients, we used highly sensitive and specific phage mediated immuno-PCR (IPCR) (Yu, Burgoon, 2007) and screened 18 paired CSF and sera from MS patients. Here, we coated plates with MS patient CSF, the corresponding patient serum, or with control 
human IgG (at IgG concentration of $1 \mu \mathrm{g} / \mathrm{ml}$ ) to Protein A plates. MS brain rAb-selected phage were incubated with the coated $\mathrm{IgG}$; bound phage were lysed and used as template DNA for IPCR. The lower the Ct value, the more phage were bound, presumably due to higher affinity for the Ab. Most of the MS IgG did not show positivises (data not shown). However, we show that phage peptides A2 (ST-196) C3 and A2 (ST-196) F4 are specific to intrathecal IgG of one MS CSF (MS03-4) but not to paired serum or human IgG controls (Fig. 3, A, C). The specificity of the intrathecal binding of MS brain rAb-selected phage peptides was further demonstrated that those phage peptides did not bind with differential affinity to an inflammatory control (chronic progressive meningoencephalitis) CSF, serum or human IgG (Fig. 3, B, D).

To demonstrate that MS brain rAb-selected phage peptides were recognised by oligoclonal IgG from this MS patient (MS 03-4), we carried out IEF immunoblots (Graner et al. , 2020, Yu, Burgoon, 2011a). Paired CSF and serum from MS patient and MS brain rAb A2 (ST 196) were resolved on agarose IEF gels and transferred to nitrocellulose membranes. The blots were probed with corresponding phage peptide A2 (ST-196) F4 followed by incubation with mouse anti-pIII antibody. A duplicate blot was probed with anti-human IgG as a positive control to reveal total oligoclonal bands. The phage peptide selected by MS brain rAb A2 (ST-196) was recognized by multiple high-density oligoclonal IgG bands in the serum, but a weaker and reduced number of bands in the paired CSF (Fig. 4).

\section{Discussion}

Despite the very high frequency and unquestioned importance of CSF oligoclonal IgG bands in MS patients, the antigenic specificity of this IgG has yet to be determined. The presence of these IgG bands has been shown to be associated with significant clinical features such as a higher level of MS disability and disease activity (Joseph, Hirst, 2009), an increased risk of a second attack of MS (Tintore et al. , 2008), and a greater conversion rate of a clinically isolated syndrome to the relapsing and remitting form of the disease (Calabrese et al. , 2012). Conversely, less severe disease is associated with absent or few oligoclonal bands in the CSF (Avasarala et al. , 2001). Thus, it is difficult to conceive that these bands represent an epiphenomenon and are not a significant disease marker and/or factor in its neuropathogenesis. Our recent publication suggests that oligoclonal IgG antibodies in MS target patient-specific peptides (Graner, Pointon, 2020). 
The approach adopted here is predicated on the importance of B-cell immunity in MS (Greenfield and Hauser, 2018) which is well accepted. Therefore, it was logical to focus on the antigenic specificity of MS brain-derived recombinant antibodies that were generated from individual clonally expanded plasma cells obtained from these brains by laser capture microdissection. The technique of panning phage displayed random peptide libraries to identify possible antigenic targets of the MS antibody response has been reported (Archelos et al. , 1998, Cortese et al. , 1996). Early studies demonstrated that recombinant antibodies derived from clonally expanded plasma cells of SSPE brains recognise their target antigens; the majority of tested rAbs bind specifically to the cause of disease, measles virus (Burgoon et al. , 2006, Burgoon, Keays, 2005, Owens et al. , 2007). The results of the current study have extended the previous studies which used phage displayed random peptide libraries to interrogate the possible binding targets of rAbs derived from single B cells of MS CSF (Yu, Gilden, 2011b, Yu, Gilden, 2006a). Using phage display library approach, we previously found that MS rAbs generated from clonal expanded single B cells of MS CSF, recognised specific peptides representing potential candidate protein antigens (Yu, Burgoon, 2011a). Further, we found that phage peptides bound specifically to IgG in MS CSF collected over time (Yu, Burgoon, 2011a). In the current study we adopted a more direct strategy using MS rAbs generated from clonally expanded single plasma cell clones obtained from MS brain lesions with well-developed laser capture microdissection techniques (Burgoon, Caldas, 2006, Owens, Ritchie, 2007).

We generated three MS brain rAbs from expanded plasma cell sequences in two MS brains: two rAbs from MS Brain A, MS rAb A1 (ST-176), and MS rAb A2 (ST-196), and one rAb from MS Brain B, MS rAb B1 (G2-160). Several of the plasma cell sequences used were extensively somatically mutated from corresponding germline alleles, indicative of previous antigen exposure. We identified 5 unique phage peptides from 25 phage peptides selected. Database searches revealed that they shared sequence homologies with Epstein-Barr nuclear antigens 4 and 6 , as well as with other human proteins. The specificity of the phage peptides to panning MS brain rAbs were confirmed by ELISA. The poly-specific nature of the positive peptides binding to rAb A1 (ST-176) was also apparent, contrasting with the monospecific nature of the peptides binding to rAb A2 (ST-196) and rAb B1 (G2-160). Variable binding affinity to the peptide clones was apparent. In addition, as previously found ( $\mathrm{Yu}, \mathrm{Burgoon}$, 2011a), all of the peptide epitopes recognised by the MS brain rAbs were determined to be 
linear as opposed to conformational. Not all conformational epitopes may be recognised by linear phage libraries (Yu, Burgoon, 2011a). This is important as B cell epitopes may be linear or conformational.

One of the most significant observations of this study was that functional antibodies can be generated from MS autopsy brain by laser capture microdissection, and the peptides identified by these brain rAbs were recognized by intrathecal IgG from other MS patients but neither paired serum IgG nor control IgG obtained from an inflammatory control. It was then important to confirm and extend this binding pattern by demonstrating that the MS brain rAbselected peptide was recognized by MS oligoclonal IgG bands. Indeed, the peptides reacted with antibody derived from multiple MS patients and supporting the notion that these peptides may have functional/disease significance. Such binding peptides have the potential to determine the identity of intrathecal IgG in MS (Yu, Gilden, 2011b).

It is important to determine the precise identity of all the peptides and the candidate proteins that have been recognised by these rAbs, although we failed to identify any common antigens based on database searches with these peptides. We recently reported that peptides identified by MS CSF IgG antibodies were patient specific (Graner, Pointon, 2020). Such information is likely to give important clues as to corresponding cognate antigens in MS brain that these binding peptides may represent, and potentially the causes of the different phenotypes of MS. In addition, the lack of common antigens to intrathecal IgG in MS may suggest that other functions of these antibodies (effector function) may be more relevant to disease pathogenesis.

It is possible that the heterogeneity of MS patients may be reflected by a corresponding heterogeneity in peptides binding to oligoclonal $\mathrm{IgG}$ as we have shown (Graner, Pointon, 2020). While the results described in this study were obtained from a small number of MS brain rAbs, RRMS and SPMS represent a diverse population of MS patients to which an expanded application of these techniques may be applied. The phage display panning technique may identify many different MS-associated peptides, and this approach offers considerable potential for enhancing our knowledge of MS pathogenesis.

\section{Acknowledgements}


The supply of MS brain recombinant antibodies was a generous gift from Dr. Mark P. Burgoon. We also thank Dr. Burgoon for his critical review of the manuscript and his dedication to MS immunology research.

This work was supported in part by Centre for Neuroscience, University of Colorado Anschutz Medical Campus, and a Pilot Research Award (PP1662) for Dr. Yu from National Multiple Sclerosis Society. The University of Colorado Genomics and Microarray Shared Resource is sponsored by Cancer Centre Support Grant (P30CA046934). Drs. Yu and Graner are supported by 5R21MH118174-02 from the NIH/NIMH.

\section{Figure legend}

Fig. 1. ELISA demonstrating binding specificities of selected phage peptides to panning MS brain rAbs. Phage peptides selected by rAb A1 (ST-176) and rAb A2 (ST-196) were amplified and used to determine specificity to panning rAbs by ELISA. Panning rAbs were coated onto wells in ELISA plates, followed by addition of corresponding phage and detected by HRP-anti M13 antibodies. A. ELISA of phage peptides identified by MS rAb A1 (ST-176) A1, C3, E2, B1 were used to determine specificity to panning rAb A1. Positive phage peptides to panning MS rAb A1 (ST-176) but not to control human IgG were observed $\mathbf{B}$. ELISA showing positive phage peptides to panning MS rAb A2 (ST-196) but not to control human IgG. C. Peptides (ST176-C3 and B1) identified by brain rAb A1 (ST-176) were polyspecific, and peptides (F4 and D2) panned by MS rAb A2 (ST-196) were specific to panning $\mathrm{rAb}$, but not to rAb from the same brain (ST-176), nor to control human IgG. D. Dose response ELISA confirming binding specificity of phage peptides A2 (ST-196) D2 and F4 to panning MS brain rAb A2 (ST-196).

\section{Fig. 2. Western blots showing that phage peptides were reactive to panning MS brain} rAbs, confirming linearity of phage peptides. Four representative phage peptides selected by 2 MS brain rAbs were separated on a gradient of 4-12\% SDS polyacrylamide gel, blotted to a PVDF membrane and incubated with corresponding MS brain rAbs. Blots of anti-pIII 
(phage protein) antibody probing with a duplicate membrane (lower panel) are shown as positive control. Brain A=rAb A2 (ST-196); Brain B=rAb B1 (G2-160).

Fig. 3. Phage IPCR showing phage peptides selected by MS brain rAb A2 (ST-196) was recognized by MS CSF intrathecal IgG, but not by paired serum IgG, nor by an inflammatory control CSF IgG. A. Phage peptide A2 (ST-196) F4 (selected by MS brain rAb A2) was recognized by a MS CSF, but not by paired serum and human IgG control. B. Phage A2 (ST-196) F4 binds with same affinity to inflammatory control CSF (IC07-2, chronic progressive meningoencephalitis), serum and human IgG control. C. Phage A2 (ST196) C3 was recognized by MS CSF, but not by paired serum and human IgG control. D. Phage A2 (ST-196) C3 binds with same affinity to inflammatory control CSF, serum and control IgG.

Fig. 4. Phage probed isoelectric focused blots demonstrate that MS brain rAb specific peptide was recognized by oligoclonal IgG bands. Paired MS CSF and serum ( $3-5 \mu \mathrm{g}$ total IgG) from one MS patient and MS brain rAb A2 (ST-196) were resolved on agarose IEF gels and transferred to nitrocellulose membranes. The blots were probed with corresponding phage peptide A2 (ST-196) F4 (10 $\left.{ }^{10} \mathrm{pfu} / \mathrm{ml}\right)$ followed by incubation with mouse anti-pIII antibody and detected by AP-anti-mouse antibody (right panel with phage probe). Duplicate blot was probed with anti-human IgG as positive controls to reveal oligoclonal bands. Peptide selected by MS brain $\mathrm{rAb}$ was recognized by multiple high-density oligoclonal $\mathrm{IgG}$ bands in serum, but weaker and reduced number of bands in the CSF (arrows).

\section{References}

Archelos JJ, Trotter J, Previtali S, Weissbrich B, Toyka KV, Hartung HP. Isolation and characterization of an oligodendrocyte precursor-derived B-cell epitope in multiple sclerosis. Ann Neurol. 1998;43:1524.

Avasarala JR, Cross AH, Trotter JL. Oligoclonal band number as a marker for prognosis in multiple sclerosis. Arch Neurol. 2001;58:2044-5.

Beseler C, Vollmer T, Graner M, Yu X. The complex relationship between oligoclonal bands, lymphocytes in the cerebrospinal fluid, and immunoglobulin $\mathrm{G}$ antibodies in multiple sclerosis: Indication of serum contribution. PLoS One. 2017;12:e0186842.

Burgoon MP, Caldas YA, Keays KM, Yu X, Gilden DH, Owens GP. Recombinant antibodies generated from both clonal and less abundant plasma cell immunoglobulin $\mathrm{G}$ sequences in subacute sclerosing panencephalitis brain are directed against measles virus. J Neurovirol. 2006;12:398-402. 
Burgoon MP, Keays KM, Owens GP, Ritchie AM, Rai PR, Cool CD, et al. Laser-capture microdissection of plasma cells from subacute sclerosing panencephalitis brain reveals intrathecal disease-relevant antibodies. Proc Natl Acad Sci U S A. 2005;102:7245-50.

Calabrese M, Poretto V, Favaretto A, Alessio S, Bernardi V, Romualdi C, et al. Cortical lesion load associates with progression of disability in multiple sclerosis. Brain : a journal of neurology.

2012;135:2952-61.

Cortese I, Tafi R, Grimaldi LM, Martino G, Nicosia A, Cortese R. Identification of peptides specific for cerebrospinal fluid antibodies in multiple sclerosis by using phage libraries. Proc Natl Acad Sci U S A. 1996;93:11063-7.

Ferreira D, Voevodskaya O, Imrell K, Stawiarz L, Spulber G, Wahlund LO, et al. Multiple sclerosis patients lacking oligoclonal bands in the cerebrospinal fluid have less global and regional brain atrophy. J Neuroimmunol. 2014;274:149-54.

Gilden DH. Infectious causes of multiple sclerosis. Lancet neurology. 2005;4:195-202.

Graner M, Pointon T, Manton S, Green M, Dennison K, Davis M, et al. Oligoclonal IgG antibodies in multiple sclerosis target patient-specific peptides. PLoS One. 2020;15:e0228883.

Greenfield AL, Hauser SL. B-cell Therapy for Multiple Sclerosis: Entering an era. Ann Neurol. 2018;83:13-26.

Joseph FG, Hirst CL, Pickersgill TP, Ben-Shlomo Y, Robertson NP, Scolding NJ. CSF oligoclonal band status informs prognosis in multiple sclerosis: a case control study of 100 patients. Journal of neurology, neurosurgery, and psychiatry. 2009;80:292-6.

Kennedy PG, Steiner I. On the possible viral aetiology of multiple sclerosis. QJM : monthly journal of the Association of Physicians. 1994;87:523-8.

Owens GP, Bennett JL, Lassmann H, O'Connor KC, Ritchie AM, Shearer A, et al. Antibodies produced by clonally expanded plasma cells in multiple sclerosis cerebrospinal fluid. Ann Neurol. 2009;65:63949.

Owens GP, Ritchie AM, Burgoon MP, Williamson RA, Corboy JR, Gilden DH. Single-cell repertoire analysis demonstrates that clonal expansion is a prominent feature of the $B$ cell response in multiple sclerosis cerebrospinal fluid. J Immunol. 2003;171:2725-33.

Owens GP, Ritchie AM, Gilden DH, Burgoon MP, Becker D, Bennett JL. Measles virus-specific plasma cells are prominent in subacute sclerosing panencephalitis CSF. Neurology. 2007;68:1815-9.

Petereit HF, Reske D. Expansion of antibody reactivity in the cerebrospinal fluid of multiple sclerosis patients - follow-up and clinical implications. Cerebrospinal Fluid Res. 2005;2:3.

Reiber $\mathrm{H}$, Ungefehr $\mathrm{S}$, Jacobi $\mathrm{C}$. The intrathecal, polyspecific and oligoclonal immune response in multiple sclerosis. Multiple sclerosis. 1998;4:111-7.

Serafini B, Rosicarelli B, Magliozzi R, Stigliano E, Aloisi F. Detection of ectopic B-cell follicles with germinal centers in the meninges of patients with secondary progressive multiple sclerosis. Brain Pathol. 2004;14:164-74.

Tintore M, Rovira A, Rio J, Tur C, Pelayo R, Nos C, et al. Do oligoclonal bands add information to MRI in first attacks of multiple sclerosis? Neurology. 2008;70:1079-83.

Yu X, Barmina O, Burgoon M, Gilden D. Identification of measles virus epitopes using an ultra-fast method of panning phage-displayed random peptide libraries. J Virol Methods. 2009;156:169-73.

Yu X, Burgoon M, Green M, Barmina O, Dennison K, Pointon T, et al. Intrathecally synthesized IgG in multiple sclerosis cerebrospinal fluid recognizes identical epitopes over time. J Neuroimmunol. 2011a;240-241:129-36.

Yu X, Burgoon MP, Shearer AJ, Gilden DH. Characterization of phage peptide interaction with antibody using phage mediated immuno-PCR. J Immunol Methods. 2007;326:33-40.

Yu X, Gilden D, Schambers L, Barmina O, Burgoon M, Bennett J, et al. Peptide reactivity between multiple sclerosis (MS) CSF IgG and recombinant antibodies generated from clonally expanded plasma cells in MS CSF. J Neuroimmunol. 2011b;233:192-203. 
Yu X, Gilden DH, Ritchie AM, Burgoon MP, Keays KM, Owens GP. Specificity of recombinant antibodies generated from multiple sclerosis cerebrospinal fluid probed with a random peptide library. J Neuroimmunol. 2006a;172:121-31.

Yu X, Owens GP, Gilden DH. Rapid and efficient identification of epitopes/mimotopes from random peptide libraries. J Immunol Methods. 2006b;316:67-74. 